Immersive Collaboration Environment

Peer-reviewed author version

DUMONT, Maarten; MAESEN, Steven; FREDERIX, Karel; RAYMAEKERS, Chris;

BEKAERT, Philippe \& VAN REETH, Frank (2010) Immersive Collaboration

Environment. In: DiNitto, E; Yahyapour, R (Ed.) TOWARDS A SERVICE-BASED

INTERNET. p. 187-188..

DOI: $10.1007 / 978-3-642-17694-4 \_16$

Handle: http://hdl.handle.net/1942/11998 


\title{
Immersive Collaboration Environment
}

\author{
Maarten Dumont, Steven Maesen, Karel Frederix, Chris Raymaekers, Philippe \\ Bekaert, and Frank van Reeth \\ Hasselt University - tUL — IBBT, \\ Expertise Centre for Digital Media \\ Wetenschapspark 2, 3590 Diepenbeek, Belgium \\ firstname.lastname@uhasselt. be
}

\begin{abstract}
Collaboration between people who are not co-located still poses many problems. In this demo, we show how to create an immersive virtual office, where people can meet each other. Different novel technologies are combined in order to reach this goal. Users can collaborate by using a multitouch interface to exchange ideas. Moreover, free-viewpoint video allows users to have eye-gaze corrected face-to-face communication. Finally, a multiprojector system is used to realize an immersive feeling of working together. We show how the integration of these technologies results in an office-of-the-future setup.
\end{abstract}

\section{Introduction}

When people are collaborating in meetings, such as brainstorming meetings, many forms of communication are used. Voice communication is very important, but people also use pieces of paper to sketch their ideas. Furthermore, nonverbal communication, such as facial expressions contribute to the meeting as it shows how people feel about the various ideas that are presented. However, when people need to collaborate over a distance, much of this information is lost. Current Computer-Supported Collaborative Work (CSCW) applications and video conferencing solutions only provide limited human interaction possibilities. We integrate multitouch interaction, free-viewpoint video and a multiprojector system to develop a more natural and immersive collaboration environment.

\section{Demo description}

The demo combines three different and novel technologies: a collaborative multitouch system, free-viewpoint video and a multiprojector system. The accompanying video can be found at http://research.edm. uhasselt.be/ mdumont/ sw2010.

\subsection{Collaborative multitouch}

Standard CSCW tools allow users to interact collaboratively. This usually boils down to sharing Windows applications. In meetings however, people tend to use 
paper to collaborate. This intuitive way of working cannot be realized using the standard CSCW tools.

In our system, users have a multitouch table at their disposal for collaboration. Such a table supports a natural way of interacting, since users can manipulate information (e.g. pictures, documents, schematics, ...) using their hands. As we have developed a framework for collaborative multitouch applications, it is possible for users to collaboratively manipulate information, each using their own separate multitouch table as if it were one virtual table. Actions on objects are distributed over the network, while users still have their own view of the information.

\section{$2.2 \quad$ Free-viewpoint video}

One major drawback of current video-conferencing systems is the fact that the user is not able to simultaneously look at the screen and into the camera. This leads to a loss of eye contact, which is an important factor in non-verbal communication.

In our setup we make use of free-viewpoint video, where the user is filmed by multiple cameras. By interpolating between the images of the cameras, every viewpoint in range of these cameras can be shown. This range includes an eyegaze corrected view that enables remote users to directly look into each other's eyes.

\subsection{Multiprojector}

A true immersive feeling can only be achieved by providing a sufficiently large projection of the remote environment. Our system uses several projectors to compose this large picture and to show a surround image of the remote site.

Manually aligning several projectors is a tedious job. We have therefore automated the calibration process. By using structured light, the projectors are automatically calibrated. This calibration process compensates for misalignment of the projectors, color difference in the images and the geometric structure of the projection surface. This allows us to project on arbitrary surfaces, including corners of a room.

\section{Integration}

These different technologies, which are on itself novel, are integrated in order to form an office-of-the-future setup. Users in this virtual office can collaborate using multitouch interaction. Meanwhile, free-viewpoint video offers natural eyegaze corrected face-to-face communication. Finally, the multiprojector system completes the illusion of full immersion into the virtual office.

\section{Acknowledgments}

This work was funded by IBBT through the Hi-Masquerade project. 